\title{
Analysis of impact between the helmet and flying rock during earthquake
}

\author{
Lan Ying ZHAO, Gang SUN ${ }^{\mathrm{a}}$, Wei Hua LI, Xuan LIU and Jing ZHU \\ National Earthquake Response Support Service, Beijing100049, China
}

\begin{abstract}
The kinematics parameters like velocity of the flying rock during an earthquake and the collision between the helmet and the flying rock is presented. Since the seismic wave could be simplified as sinusoid individually in the horizontal direction and the vertical direction, the velocity of the flying rock is calculated separately with the acceleration and the frequency in the correspondingly orientation. Then, theoretical model of the collision process include velocity and the impact force is actualized based on the analysis of cases not only the impact between the rock and the helmet lonely but also the rock and the helmet well-worn on the rescuer's head.
\end{abstract}

\section{Introduction}

Except for causing a large number of people's lives and property losses, severe earthquakes also trigger a lot of geological disasters or secondary disasters such as the landslides, debris flows, barrier lakes, tsunami, flood, etc. For earthquake rescue missions, the rolling and flying rocks from the loose rock mass structure take serious threat to the lifeline engineering, vehicles and rescuers and affect the rescue mission. Meanwhile, severe earthquakes cause a large number of constructions collapsed and damaged thus unable to stay. Many concrete components of the building ruins explode outside and sometimes fall towards the earth which will hurt somebody or even cause somebody to die if one is just in the position[1,2].

The movement of rolling rocks during earthquakes had been an issue and many literatures studied it. The motion types were divided to several kinds like slipping, rolling, free-faller, projectile, etc and series equations were proposed in order to calculate track and speed of the rolling rocks[3,4]. Xiao Feng analyzed selected samples of perilous sector with potential rockfall and the farthest, the nearest distance of the rolling rocks [5]. Tang Hongmei proposed the reasons of rolling rock and obtained the kinematic trajectory equation using the collision theory, instantaneous friction role theory[6].Factors influenced the movement of the rolling rocks were analyzed and the mainly factor is sequential the slope and slope conditions, the shape of the rolling stones, and the rock quality according to their influence[7-9].The motion feature, the energy absorption way and the harm scope were analyzed taking Wenchuan earthquake as an example[10]. Based on the statistical analysis of the 399 collapsed stones profiles, the slope angle was respective 56 degrees, 39.6 degrees, 26 degrees to form different motion type of the rolling rock and good linear relationship existed not only between the maximum movement distance and slope but also between the spread of the rock and the slope[11].

There were less literatures about the impact force than the movement of the rolling rocks and mainly concentrated on engineering protection demand. The Hertz elastic collision theory and the impulse theorem was the base of the calculation and there were the Swiss formula, formula of Japan, Australian formula, roadbed code formula, Yang Qixin formula as typical algorithm [12-16]. Yuan Jinke compared the above algorithms and introduced his method to calculate the impact force [17].

For the kinematics and impact force of the flying rocks, there were few research and mainly about the engineering blasting. Chen Kang of the blasting flying rock based on the data of the Three Gorges Dam using the similarity theory[18]. Wu Chunping obtained the distance equation suitable for one same blasting project using the dimensional analysis method[19].In addition, the BP neural network model and the grey Elamn neural network were also applied to calculate the distance of flying rocks[20, 21]. However, there is little research about lying rocks caused by earthquakes. In this paper, the speed of the flying rock is analyzed with the acceleration and the frequency of the seismic wave in three orientations. Besides, the collision process between the rock and the earthquake rescuer helmet is presented and the calculate module of the collision is carried out.

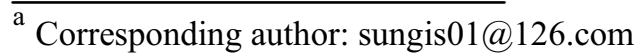




\section{The speed of the flying rocks}

\subsection{Model and setting}

Considering one rock flies out from the original position with the affection of the seismic wave in the horizontal direction and the vertical direction after an earthquake and it will get certain speed through the seismic wave. As known, seismic wave could be regarded as combination of a series of sinusoid, the affection of the seismic wave could be decompose as the combined action of the series of sinusoid. The wave records of the seismic station are divided to three parts as E-W, N-S, U-D and represent the seismic wave of east-west, north-south and vertical direction. We simplified each part of the seismic wave to be a sinusoid and influenced the rock on time $\mathrm{t}$, and the rock flied out with the affection as shown in Fig.1. The speed of the flying rock is the vector superposition of the speed in three directions, so we can calculate affect of each part and then synthesize the affects to obtain the final result. In order to analyze the affect, there are parameters setting as shown in Fig. 2 .

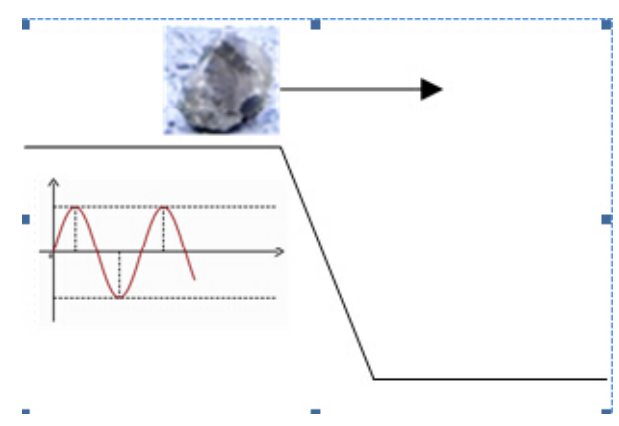

Figure1. The flying rock affected by the seismic wave

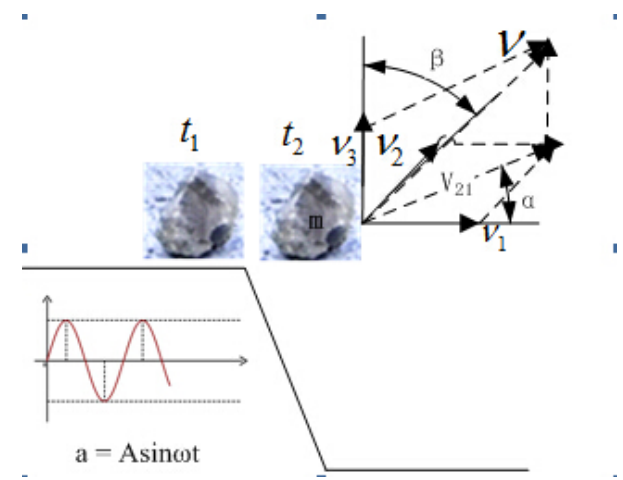

Figure 2. The parameters setting

$t_{1}$ - the time of seismic wave affect the rock

$t_{2}$ - the time of rock fly from the original position

$v_{1}$ - the speed in E-W direction

$v_{2}$ - the speed in N-S direction

$v_{3}$ - the speed in U-D direction

$v_{21}$ - the vector superposition of $v_{1}$ and $v_{2}$

$\alpha$ - the intersection angle between $v_{1}$ and $v_{2}$

$\beta$ - the intersection angle between $v_{21}$ and $v_{3}$ $\mathrm{m}$-the quality of flying rock

$\mathrm{a}=$ Asin $\omega$ t-the expression of the seismic wave

\subsection{The horizontal speed calculation of flying rock}

The speed in E-W and N-S direction is similar and both belong to the horizontal speed and the E-W speed is used to expound the calculation here. With the influence of the E-W part of the seismic wave, the flying rock could get the starting speed:

$v_{1}=\int_{t_{1}}^{t} A_{1} \sin \omega_{1} t d t=A_{1}\left(\frac{\cos \omega_{1} t_{1}}{\omega_{1}}-\frac{\cos \omega_{1} t}{\omega_{1}}\right)$

and the maximum of $v_{1}$ is:

$v_{1 m}=\frac{2 A_{1}}{\omega_{1}}$

There will be air friction during the flying and we consider the air friction is opposite with the flying direction and it is a linear relation with them, the linear correlation coefficient $\mathrm{k}$ is:

$f=-k v$

During the flying of the rock, with the barriers of air friction,the speed of the rock is :

$\frac{d v_{1}}{d t}=-\frac{k v_{1}}{m}$
$v_{1}=c_{1} e^{-\frac{k t}{m}}$

and the constant

$$
c_{1}=\frac{2 A_{1}}{\omega_{1}} e^{\frac{k t_{2}}{m}}
$$

so the E-W speed :

$v_{1}=\frac{2 A_{1}}{\omega_{1}} e^{\frac{k t_{2}}{m}} e^{-\frac{k t}{m}}$

and similarly, the N-S speed

$v_{2}=\frac{2 A_{2}}{\omega_{2}} e^{\frac{k t_{2}}{m}} e^{-\frac{k t}{m}}$

Furthermore, there is the vector superposition of $v_{1}$ and $v_{2}$ :

$v_{21}=\sqrt{v_{1}^{2}+v_{2}^{2}}=2 \sqrt{\left(\frac{A_{1}}{\omega_{1}}\right)^{2}+\left(\frac{A_{2}}{\omega_{2}}\right)^{2}} e^{\frac{k t_{2}}{m}} e^{-\frac{k t}{m}}$ 


$$
\alpha=\arctan \frac{v_{2}}{v_{1}}=\arctan \frac{A_{2} \omega_{1}}{A_{1} \omega_{2}}
$$

\subsection{The vertical speed calculation of flying rock}

The difference between the horizontal and vertical direction is the gravity. During the flying, the rock will barge up against both of the air friction and the gravity and there will be equation similar with the equation (4):

$$
\begin{aligned}
& \frac{d v_{3}}{d t}=-\frac{k v_{3}}{m}-g \\
& v_{3}=\left(\frac{m g}{k}+\frac{2 A_{3}}{\omega_{3}}\right) e^{\frac{k t_{2}}{m}} e^{-\frac{k t}{m}}-\frac{m g}{k}
\end{aligned}
$$

Similarly, there is the vector superposition of $v_{21}$ and $v_{3}$, we can get the speed of the flying rock:

$$
\begin{aligned}
& v=\sqrt{v_{21}{ }^{2}+v_{3}{ }^{2}} \\
& \beta=\arctan \frac{v_{21}}{v_{3}}
\end{aligned}
$$

\section{Impact analysis}

\subsection{Type of collision}

The collision happens in short time with big impulse when the flying rock impacts the helmet and there will be three types according to the displacement in the collision.

Firstly, if the rescue helmet is not fixed or worn with rescuer, it will obtain a movement after the collision and move to a certain direction with a velocity. This collision process can be simplified as the elastic collision that there will be no displacement and plastic deformation at the contact point.

Secondly, if the rescue helmet is fixed or well worn with the rescuer and the quality of the flying rock is not too big to affect the movement of rescuer head, which means there is no displacement or little displacement that can be ignored. This collision process can be simplified as the nonelastic collision with no displacement at the contact point.

Thirdly, if the rescue helmet is fixed or well worn with the rescuer and the quality of the flying rock is big enough to affect the movement of rescuer head, since the dimensions of flying rock is smaller than the rescuer height, the helmet will obtain angular rotation instead of little linear displacement after the collision. This collision process can be simplified as the nonelastic collision that there will be rotation movement at the contact point.

\subsection{The elastic collision}

There are assumptions as follow to analyze the elastic collision between the flying rock and the helmet:
1) The affection of the gravity force and the elastic force is ignored since they are fewer compared with the impact force.

2) Because the collision process is very short time, the flying rock and the helmet has no linear displacement during the collision and they will move with new velocity after the collision.

3) The flying rock and the helmet is regarded as one system and there is no outer energy or force influencing the system, so there is conversation of energy during the whole collision.

Based on the above assumption, the collision could be modeled as two rigid bodies collision as shown in Fig.3.

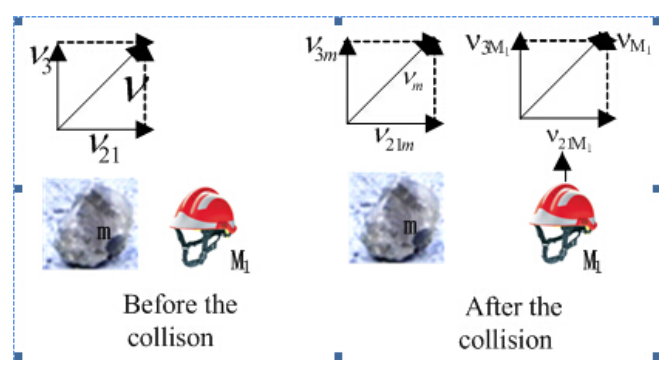

Figure 3. The model of the elastic collision between the rock and the helmet

$\mathrm{M}_{1}$-the quality of rescue helmet

$\mathrm{V}_{\mathrm{m}}$ - the speed of flying rock after the collision

$\mathrm{V}_{\mathrm{M} 1}$ - the speed of helmet after the collision

If there is coefficient of restitution as $\mathrm{k}_{\mathrm{m}}$, then

$\mathrm{k}_{\mathrm{m}}=\frac{\mathrm{I}_{\mathrm{M}_{1}}}{\mathrm{I}_{\mathrm{m}}}=\frac{\mathrm{v}_{\mathrm{M}_{1}}-\mathrm{v}_{\mathrm{m}}}{\mathrm{v}}$

According to the momentum conservation theorem, there has equation about the velocity before and after the collision:

$\mathrm{mv}=\mathrm{m} v_{\mathrm{m}}+\mathrm{Mv}$

With the equations (15) and (16), we can obtain the velocity of the flying rock and the helmet:

$$
\begin{aligned}
& v_{\mathrm{m}}=v-\left(1+\mathrm{k}_{\mathrm{m}}\right) \frac{\mathrm{m}}{\mathrm{m}+\mathrm{M}_{1}} \mathrm{v} \\
& v_{\mathrm{M}_{1}}=\left(1+\mathrm{k}_{\mathrm{m}}\right) \frac{\mathrm{m}}{\mathrm{m}+\mathrm{M}_{1}} \mathrm{v}
\end{aligned}
$$

Moreover, according to the impulse theorem, there will be:

$m v_{m}-m v=\int_{0}^{t} F d t$

$\mathrm{M}_{1} v_{\mathrm{M}_{1}}=\int_{0}^{\mathrm{t}} \mathrm{Fdt}$

With the equations (19) and (20), we can obtain the impact force during the collision: 


$$
\mathrm{F}=\frac{\mathrm{M}_{1} v_{\mathrm{M}_{1}}}{\Delta \mathrm{t}}=\frac{\mathrm{m} v_{\mathrm{m}}-\mathrm{mv}}{\Delta \mathrm{t}}
$$

The energy loss during the collision:

$$
\begin{aligned}
\Delta \mathrm{T}= & \frac{1}{2} \mathrm{mv}_{\mathrm{m}}{ }^{2}+\frac{1}{2} \mathrm{M}_{1} \mathrm{v}_{\mathrm{M}_{1}}{ }^{2}-\frac{1}{2} \mathrm{~m} v^{2} \\
& =\frac{\mathrm{mM}_{1}}{2\left(\mathrm{~m}+\mathrm{M}_{1}\right)}\left(1-\mathrm{k}_{\mathrm{m}}{ }^{2}\right) v^{2}
\end{aligned}
$$

\subsection{The nonelastic collision with no displacement}

In this type of collision, the flying rock, the helmet and the rescuer head are regarded as one system and the collision could be modeled as shown in Fig.4. Since there is no outer energy or force influencing the system, the conversation of energy is suitable during the whole collision and there is:

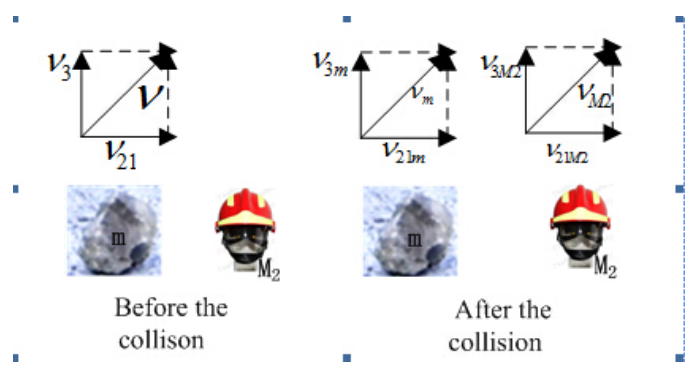

Figure 4. The model of the nonelastic collision with no displacement

$$
\iint_{s} F \mu d s+\frac{L F^{2}}{2 E A}+W_{2}=\frac{1}{2} M_{2} v_{M 2}^{2}+\frac{1}{2} \iiint_{V} e_{i j} X_{i j} d V
$$

For the left side of the equation (23), the first part is the energy of the impact force.

The second part is the elastic deformation energy $\mathrm{W}_{1}$ :

$W_{1}=\frac{L F^{2}}{2 E A}$

$\mathrm{L}$ - the height of the head-helmet combination

E - the elastic modulus of the head-helmet combination

A - the sectional area of the contact point

The third part is the energy consumption of the vibration of the head-helmet combination $\mathrm{W}_{2}$ :

$\mathrm{W}_{2}=\mathrm{k}_{\mathrm{M}_{2}} \int_{0}^{\mathrm{L}} \mathrm{M}_{2} \mathrm{D}(\mathrm{x}) \mathrm{dx}=\mathrm{k}_{\mathrm{M}_{2}} \mathrm{M}_{2} \mathrm{D}^{2}$

$\mathrm{K}_{\mathrm{M} 2}$ - the natural frequency of the head-helmet combination

$\mathrm{D}(\mathrm{x})$ - the vibration amplitude of the head-helmet combination

$\mathrm{M}_{2}$ - the quality of the head-helmet combination
For the right side of the equation (23), the first part is the kinetic energy of the head-helmet combination $\mathrm{W}_{3}$ :

$\mathrm{W}_{3}=\frac{1}{2} \mathrm{M}_{2} \mathrm{v}_{\mathrm{M}_{2}}^{2}$

The second part is the elastic deformation energy of the head-helmet combination $\mathrm{W}_{4}$ :

$W_{4}=\frac{1}{2} \iiint_{V} e_{i j} X_{i j} d V$

$\mathrm{e}_{\mathrm{ij}}$ - the dynamic stress of the head-helmet combination $\mathrm{X}_{\mathrm{ij}}$ - the dynamic strain of the head-helmet combination

\subsection{The nonelastic collision with rotation movement}

In this type of collision, the flying rock, the helmet and the rescuer are regarded as one system and the rescuer may have fixed axis rotation with the foot as the pivot axis and the rescuer height as the shaft length and the collision could be modeled as shown in Fig.5.
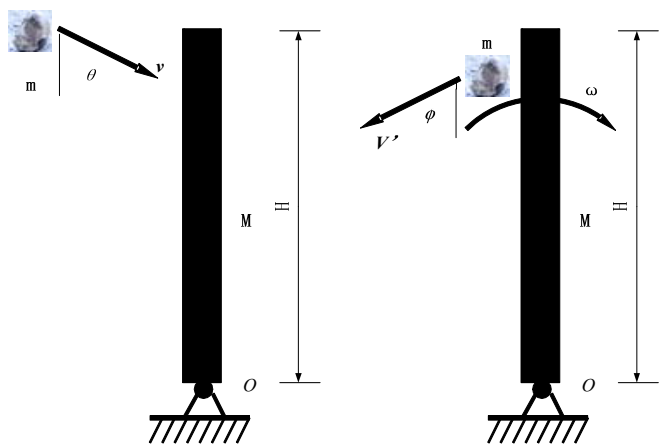

Figure 5. The model of the nonelastic collision with rotation movement

$V$ _the velocity of the flying rock before the collision

$V^{\prime}$ - the velocity of the flying rock after the collision

$\theta$-angle between $\mathrm{V}$ and the vertical

$\phi$-angle between $v^{\prime}$ and the vertical

$\mathrm{M}$ - the quality of rescuer and helmet

$\mathrm{H}$ - the height of rescuer

$\omega$ - the rotational angular velocity of rescuer

There is no outer energy or force influencing the system, so the system momentum is conserved and there is:

$m v \sin \theta \bullet H=J_{0} \omega+m v^{\prime} \sin \phi \bullet H$

For the left side of the equation (24), there is

$J_{0}=\frac{1}{3} M H^{2}$

$v^{\prime}=\omega H$ 
and the rotational angular velocity of rescuer is:

$\omega=\frac{3 m}{(3 m+M) H} \frac{\sin \theta}{\sin \phi} v$

According to the theorem of conservation of momentum, in the collision process there is:

$m v^{\prime} \sin \phi+M \omega H-m v \sin \theta=I_{x}$

$0-m v^{\prime} \cos \phi=I_{y}$

Take the equation (25) into the equation (26) and (27), then

$I_{x}=\left(\frac{3 m}{3 m+M}+\frac{3 m}{3 m+M} \frac{1}{\sin \phi}\right) m v \sin \theta$

$I_{y}=-\frac{3 m}{3 m+M} \operatorname{ctg} \phi \bullet m v \sin \theta$

If the impact time is signed as $\Delta t$, the impact force during the collision could be calculated as follow:

$F_{x}=\frac{I_{x}}{\Delta t}$

$F_{y}=\frac{I_{y}}{\Delta t}$

There is two special situation when $\theta$ is separately 0 and 90 degrees. When $\theta$ is 0 degree which means the flying rock has free-fall movement, there is no rotation and only displacement in the vertical direction like the elastic collision, the velocity of the rescuer and the flying rock will be:

$v^{\prime}=v-(1+k) \frac{m}{m+M} v$

$v_{M}=(1+k) \frac{m}{m+M} v$

and the impact force will be:

$F=\frac{M v_{M}}{\Delta t}=\frac{m v_{m}-m v}{\Delta t}$

When $\theta$ is 90 degrees which means the flying rock has horizontal movement and attack the side of rescuer, there is no displacement and only rotation, the rotational angular velocity and the impulse of rescuer will be:

$\omega=\frac{3 m}{(3 m+M) H} v$

$I_{x}=\frac{6 m}{3 m+M} m v$
$I_{y}=0$

and the impact force will be:

$F=F_{x}=\frac{6 m^{2} v}{(3 m+M) \Delta t}$

\section{Conclusion}

Focused on the flying rock caused by the earthquakes, the velocity in the horizontal and vertical orientation is calculated with the simplified sinusoid model of seismic wave and the speed could be obtained through the vector superposition of velocity in the different directions. When the flying rock shocks the helmet or the rescuer, three types of collision is introduced and with the analysis one by one, the kinematics parameters and the impact force is calculated in the paper using the theorem of momentum, impulse and angular momentum.

\section{Acknowledgment}

This work was supported by the Science for Earthquake Resilience of China Earthquake Administration (No.XH16045Y, No.XH16046).

\section{References}

Here are some examples:

1. X.P.Tian, J.Z.Sun, Q.Chen, L.P.Liu, A.P.Tian, J. Eng. Geol.17,3(2009).

2. Q.Xu, R.Q.Huang, J. Eng. Geol.16,6(2008).

3. Q.Lv, H.Y.Sun, S.K.Zhai, H.B.Wang, Y.Q.Shang, J. Nat. Disaster.12,2(2003).

4. H.Q.Yang,X.P.Zhou, Rock Soil Mech.30,11(2009).

5. X.Feng,J.Guo,J.Liu, Subgrade Eng.6(2013).

6. H.M.Tang, P.Y.Yi, J. Chongqing Jianzhu U. 25,1 (2003).

7. R.Q.Huang, W.H.Liu, J. Rock Mech.\& Eng. 28,5 (2009).

8. R.Q.Huang, W.H.Liu, J.P.Zhou, X.J.Pei, J. Geo. E. 29,9(2007).

9. X.J.Pei, R.Q.Huang, Z.Pei, X.J.Dong, J. Eng. Geol. 19,4(2011).

10. B.L.Li, X.J.Pei, Q.Cheng, S.G.Li, Gansu Water Resources And Hydropower Technology .46,2(2010).

11. Q.Cheng, S.R.Su, Rock Soil Mech. 35,3(2014).

12. S. Kawahara, T. Muro, J. Terramechanics. 43,3(2006).

13. V. Labiouse, F. Descoeudres, S. Montani, STRUCT ENG INT.6,3(1996).

14. B. Pichler, C. Hellmich, and H. A. Mang, INT J IMPACT ENG.31,5(2005).

15. JTG D30-2004, Specifications for Design of Highway Subgrades (China Communication Press,2006).

16. Q.X.Yang,B.S.Guan, J Chin Railway Soc., 1(1996). 
17. J.K.Yuan, R.Q.Huang, X.J.Pei, Rock Soil Mech. 35,1(2014).

18. K.Cheng, C.S.Zhang, J. Rock Mech.\& Eng. 19,4 (2000).

19. C.P.Wu,L.S.Liu,J.L.Dou,G.Q.Zhang, Eng. Blasting, 18,2(2012).

20. J.H.Chen,Y.Peng,S.L.Wu, Blasting,32,1(2015).

21. Q.Liu,G.Q.Zhang,C.P.Wu,T.J.Tao, Blasting,30,1 (2013). 